\title{
Virtual Determination of Blood Urea Obtained from Rats Fed Normo or Hypoprotein Diet
}

\author{
Trindade, V.M.T; Nunes, V.V; Zanatta, G.; Matté, C.; Salbego, C.G.
}

Departamento de Bioquímica, Instituto de Ciências Básicas da Saúde, Universidade Federal do Rio Grande do Sul, Porto Alegre, RS, Brazil.

Introduction: The urea cycle represents a metabolic pathway of great relevance to the nitrogen homeostasis of mammals. In addition, the metabolites involved in their reactions play a key role in the pathogenesis of some diseases and clinical situations. Therefore, it is essential to health professionals understand this metabolic pathway. Objectives: This learning object aims to illustrate the biochemical reactions of urea cycle, contextualizing clinically the main metabolites involved, as well as to present an interactive virtual urea determination in blood serum of rats fed with normo and hypoprotein diet. Materials and Methods: This object was developed with HTML5 and contains: a) hypertexts, images explaining the urea cycle, its importance and related pathologies; b) animations of the intracellular chemical reactions involved in the route of urea synthesis; c) animations simulating laboratory activities. Results and Discussion: The animated simulation of a standard urea curve, followed by virtual determination of urea in blood serum samples were developed, associated with some interactive questions to measure the user's learning. The object was assessed by undergraduate students of Basic Biochemistry. The navigation features, design and interactivity have been evaluated as excellent by about $85 \%$ of them. Conclusion: This learning object can be considered an adequate teaching resource as well as an innovative support in the construction of theoretical and practical knowledge of Biochemistry for students of health area.

Keywords: urea cycle, learning object, standard curve simulation Support: Secretaria de Educação à Distância-UFRGS 